\title{
A COMPREENSÃO ESCRITA DE ABSTRACTS DE ARTIGOS DE PESQUISA EM ENGENHARIA FLORESTAL: UMA PROPOSTA BASEADA EM GÊNERO E TAREFA
}

The Written Comprehension of Abstracts in Forest Engineering: a Proposal Based on Genre and Task

Fernanda Beatriz Caricari MORAIS, PUC-SP ${ }^{1}$

RESUMO: Este trabalho tem por objetivo apresentar uma proposta de compreensão escrita (leitura) de abstracts de artigos da área de Engenharia Florestal visando atender as necessidades dos alunos ingressantes dessa área, em especial, os alunos da pós-graduação, que possuem um conhecimento básico da Língua Inglesa. As atividades propostas seguem a concepção de gênero como um evento comunicativo e a noção de tarefa, propondo, assim, um trabalho maior com o mundo real e com o uso de dados lingüísticos autênticos.

PALAVRAS-CHAVE: Ensino de Gêneros; Ensino-Aprendizagem de Línguas para Fins Específicos, Ensino Baseado em Tarefas.

ABSTRACT: This study aims to show a proposal for reading abstracts in Forest Engineering in order to attend the student's needs, specially, postgraduate students that have basic knowledge of English language. The proposed activities are based on the conception of genre as a communicative event and the notion of task to propose work in the real world with the use of authentic linguistic data.

KEYWORDS: Genres; Language for Specific Purposes; Task-Based Approach.

\section{Introdução}

Este artigo se insere na área de elaboração de materiais para o ensino de línguas para fins específicos e foi motivado por minha atuação, em uma escola de línguas, como professora de Inglês de alunos da pós-graduação de Engenharia Florestal. Os alunos procuravam esse curso para se prepararem para o exame de proficiência de língua estrangeira de uma universidade pública no interior do estado de São Paulo.

O exame de proficiência, com duração de 3 horas, consiste em um artigo da área de estudo com perguntas relativas à compreensão escrita. Para minha surpresa, os artigos

\footnotetext{
${ }^{1}$ Possui Graduação em Letras - Inglês/Português pela Pontifícia Universidade Católica de Campinas (PUCCamp), Mestrado em Linguística Aplicada e Estudos da Linguagem Pontifícia Universidade Católica de São Paulo (PUC-SP). Atualmente é Doutoranda dessemesmo programa.
} 
eram lidos com muita dificuldade, mesmo se tratando de textos da área de estudos dos alunos. A dificuldade não era o vocabulário técnico, como nomes de espécies de plantas ou de procedimentos metodológicos na Língua Inglesa, mas sim os outros itens léxicogramaticais. Como hipótese inicial, acreditava que essa dificuldade estava relacionada à falta de conhecimento da língua alvo, pois mesmo quando os alunos dominavam o vocabulário técnico, a falta de conhecimento na Língua Inglesa comprometia toda a compreensão do texto.

O primeiro desafio que os alunos relatavam na leitura de um artigo era o abstract, um gênero muito comum na comunidade acadêmica, mas pouco conhecido por eles. Segundo seus relatos, na graduação, eles haviam tido pouco contato com abstracts, pois liam pouco ou nenhum artigo científico.

Pensando nas necessidades desses alunos, pretendo, neste artigo, propor uma unidade baseada na concepção de gênero e tarefa, pois possibilita o trabalho com a linguagem em uso, levando em conta o contexto social, os participantes da comunicação e, principalmente, o seu objetivo comunicativo.

Abordarei, primeiramente, a proposta de ensino baseado em tarefas, que envolve a troca de significados na língua alvo. Em seguida, discutirei a concepção de gêneros textuais, exemplificarei a estrutura e organização retórica do abstract e, por fim, apresentarei a proposta da unidade baseada nos pressupostos teóricos mencionados.

\section{O ensino baseado em tarefas}

Leffa (2003) discute que atualmente têm-se privilegiado o ensino baseado na tarefa, pois, dessa forma, há uma maior preocupação com o mundo real e com o uso de dados lingüísticos autênticos.

Segundo os autores Willis e Willis (1996), o ensino baseado em tarefas envolve a especificação e não a sequiência de itens lingüísticos, mas a sequiência de tarefas comunicativas, cumpridas na língua alvo. Por isso, a noção central das tarefas comunicativas é a troca de significados.

A tarefa, para esses autores, tem por objetivo encorajar os aprendizes para o desenvolvimento de um sistema de significados. Ellis (2003) aponta a importância do papel da tarefa tanto no ensino de segunda língua como na pedagogia e nas pesquisas. $\mathrm{O}$ 
autor entende tarefas como: "atividades com foco no significado e na linguagem em uso" As tarefas, também, requerem de seus participantes que desempenhem funções como usuários da língua, envolvendo-os nas atividades de mundo real. Dessa maneira, requer primeiramente do aprendiz atuar como um usuário da língua alvo.

O autor chama a atenção para a autenticidade situacional, que, para ele, é de suma importância para atingir o objetivo da tarefa: "a definição de tarefa, discutida neste livro, inclui tarefas retiradas de situações autênticas e/ou que busquem atingir a autenticidade internacional ${ }^{3}$. (Ellis, 2003, p. 6).

Para Ellis (2003) a tarefa precisa corresponder a uma atividade do mundo real. O autor (2003, p. 4) apresenta, em seu livro, várias definições de tarefa, entre elas a de Nunan (1989):

\begin{abstract}
"Uma tarefa comunicativa é "uma parte do trabalho de sala de aula que envolve os alunos na compreensão, manipulação, produção ou interação na língua alvo enquanto a atenção deles está focada, primeiramente, no significado e depois na forma".
\end{abstract}

Tomlinson (1998) acredita que é relativamente fácil mostrar os alunos de ESP (English for Specific Purposes) que os pontos a serem ensinados são relevantes e úteis, relacionando-os com os interesses da vida real dos aprendizes (real-life tasks) com o que eles precisam na língua alvo. A relevância e utilidade podem ser apresentadas pela relação de pontos a serem ensinados que sejam de interesses deles e o professor pode propor desafios em tarefas de sala de aula, discutindo com eles maneiras que podem facilitar os resultados (outcomes) por eles desejados.

Ainda sobre ESP, encontra-se uma citação de Bhatia (1997, p. 318) que discute a importância do ensino contextualizado, com foco no significado:

No ensino de inglês, especialmente no ensino de inglês para fins específicos, o aspecto mais importante do ensino é a aquisição da habilidade de usar a língua no contexto em que o aprendiz é ou fará parte dele. Aprender uma língua envolve, portanto, um processo de contextualização ${ }^{5}$.

\footnotetext{
${ }^{2}$ Original: "activities that call for primarily meaning-focused language use".

3 Original: "The definition of task that informs this book will include tasks that are both situationally authentic and/or seek to achieve international autenticy".

${ }^{4}$ Original: "A communicative task is 'a piece of classroom work which involves learners in comprehending, manipulating, producing or interacting in the target language while their attention is principally focused on meaning rather than form".

5 Original: "In the teaching of English, especially ESP, the most important aspect of learning is the acquisition of the ability to use language in the context in which the learner is or is going to be part of. Learning a language, therefore, essentially involves a process of contextualization".
} 
Uma alternativa proposta por Ellis (2003), utilizada como base nesse artigo, é a classificação de tarefas retoricamente utilizando a concepção de gênero, definido por Swales (1990), como uma classe de eventos comunicativos que partilham muitos propósitos comunicativos. Exemplares de um determinado gênero não compartilham somente a estrutura, mas sim, o propósito. Swales sugere que o veículo pedagógico ideal para ensinar gêneros é a tarefa, pois a ela deve-se incorporar um propósito comunicativo autêntico, para assim tratar um texto como um gênero baseado em tarefa (genre-based task). Isso requer o estabelecimento da situação sócio-cultural da tarefa, identificando em quais veículos determinado gênero pode ser encontrado, quem são os leitores e autores dele e como ele é organizado. A seguir, tratarei mais sobre gêneros textuais, conforme autores da área.

\section{Gêneros textuais}

Para este artigo procuro adotar uma proposta de trabalho que fundamenta o ensino de línguas com base no conceito de gêneros textuais. De acordo com Ramos e Santos (2004), a análise baseada em gêneros possibilita uma visão do texto, pois se insere no contexto social em que acontece, levando em conta a situação, os seus participantes e seu objetivo comunicativo. Swales (1990) discute a concepção de gênero como eventos comunicativos, com propósitos compartilhados por membros de uma determinada comunidade discursiva. Dessa forma, os gêneros, nessa perspectiva, podem ser entendidos como:

classes de eventos comunicativos tipicamente com características de estabilidade, nome reconhecido e assim por diante. Eventos comunicativos consistem nos textos propriamente, nos tipos de gêneros (falado, escrito ou uma combinação desses dois), partilhando características ligadas ao gênero, ao seu papel e ao seu contexto ${ }^{6}$. (SWALES, 1990, p. 09-10).

\footnotetext{
${ }^{6}$ Original: “(...) classes of communicative events which typically possess features of stability, name recognition and so on. Genre-type communicative events (and perhaps others) consist of texts themselves (spoken, written or a combination) plus encoding and decoding procedures as moderated by genre-related aspects of text-role and text-environment".
} 
O propósito comunicativo que motiva a realização do gênero, é reconhecido por uma comunidade discursiva que o molda e o transforma, como apontam Swales (1990) e Bhatia (1997). Dessa forma, podemos entender e interpretar o significado das escolhas lingüísticas de um texto a partir da caracterização do contexto de situação, dos participantes do evento e da inter-relação entre contexto e participantes. Como aponta Eggins (1994), o conceito de gênero está relacionado com o contexto de cultura, em oposição ao contexto de situação, que está relacionado ao registro que se refere ao contexto de situação, ou seja, descreve o impacto das dimensões do contexto imediato da situação de um evento no modo como a linguagem é utilizada.

Halliday e Hasan (1989) discutem a importância de não considerar o texto apenas um produto, mas sim, levar em consideração o contexto de cultura e o contexto de situação em que os textos são produzidos. O gênero é social, pois o usuário infere os significados dos textos que tem contato a partir do meio social e cultural em que vive.

A análise de um gênero para propósitos educacionais, segundo Ramos e Santos (2004), envolve a identificação:

- Da comunidade discursiva, que pode ser entendida como o grupo de pessoas que exercem regularmente uma atividade social e/ou ocupacional comum. Está relacionada com o contexto de cultura;

- Do propósito comunicativo, que é o "para quê" da atividade social; que conduz ao item a seguir;

- A organização retórica da mensagem, que se faz por meio de levantamento de blocos retóricos que desempenham a função de sinalizar o conteúdo informacional e parte do propósito comunicativo. Swales (1990) chamou esses blocos de movimentos e passos, nomenclatura que será utilizada, nesse artigo, para analisar o gênero abstract, mostrando como eles podem contribuir para uma compreensão mais profunda dos gêneros de modo geral, em especial, do gênero analisado neste trabalho.

Para Martin (1992), o gênero, o registro e a linguagem se desenvolvem em camadas, pois os primeiros, por serem abstratos, materializam-se no texto através do terceiro. O autor, em seus trabalhos, dá especial importância aos elementos léxicogramaticais relacionados com a estrutura organizacional.

Conforme apontam Eggins e Martin (1997), são os elementos léxico-gramaticais que realizam as variáveis (gênero e registro) e colaboram na identificação do propósito e 
da estrutura textual. Por isso, os textos passam a ser vistos como construções semióticas de significados socialmente construídos. Ramos (2004) explica que os gêneros não são construções neutras da realidade social, mas construções carregadas de ideologias. Cabe, portanto, ao analista explicar de que modo os textos servem a interesses diversos.

Swales (1990) investigou padrões organizacionais e retóricos responsáveis pela organização de um texto. Ele nomeia os blocos retóricos funcionais de movimentos e passos, conforme dito anteriormente, possibilitando o conhecimento da organização retórica do gênero estudado, introdução de artigos de pesquisa em inglês, em seus aspectos obrigatórios e opcionais. Segundo Ramos (2004), esse estudo oferece um modelo metodológico de descrição de gêneros com relevantes implicações pedagógicas para o campo de ensino-aprendizagem instrumental de Inglês, pois possibilitar uma compreensão escrita mais eficaz aos aprendizes, não vendo o texto como unidades gramaticais, mas unidades de significados.

Bhatia (1997), baseado em Swales (1990), inclui a possibilidade de mais de um propósito comunicativo reconhecido pelos seus usuários. De acordo com Bhatia (1993), o aspecto psicológico ou cognitivo na construção do gênero tem papel relevante para que o conceito de gênero possa ser visto como um processo social dinâmico ao invés de estático. Ao comentar a análise de um gênero, o autor indica as seguintes orientações: o aspecto sociológico, fatores provenientes da realidade social (contexto em que se processa a negociação, os papéis sociais dos participantes, os propósitos dessa interação, as preferências profissionais dos participantes, as organizacionais e as restrições culturais) e o aspecto psicológico, que são as estratégias pelas quais o gênero é produzido.

Portanto, resumindo as concepções apresentadas, o gênero é entendido, nesse artigo, como um processo social dinâmico, com um ou mais propósitos comunicativos, altamente estruturado e convencionalizado, reconhecido e mutuamente compreendido pelos membros da comunidade em que ele ocorre. $O$ gênero opera não só dentro de um espaço textual, mas também dentro de um espaço discursivo, estratégico e sócio cultural.

$\mathrm{O}$ aprendiz, que estuda a língua alvo através de gêneros, pode adquirir maior percepção de que escolhas lingüísticas devem ser feitas tendo como pressuposto o contexto cultural (gênero) e situacional (registro) em que se encontra. Para Vian Jr. (2003), o ensino contextualizado deve situar o aprendiz e conscientizá-lo do papel social da linguagem e que ele, como usuário da língua, terá escolhas que refletirão o contexto situacional e cultural em que se encontra. 
Segue, no item a seguir, a análise de um exemplar do gênero abstract da área de Engenharia Florestal com base nos pressupostos teóricos discutidos anteriormente, com foco nas características desse gênero, sua organização em movimentos e passos, bem como suas escolhas léxico-gramaticais.

\section{O abstract}

Neste item, apresentarei as principais características do gênero abstract de artigos de pesquisa da área de Engenharia Florestal, tais como as características do contexto de cultura e de situação (o propósito, os participantes, a circulação do gênero), a organização retórica e as características léxico-gramaticais. Para isso, dez exemplares do gênero foram coletados das principais revistas científicas da área de Engenharia Florestal do país. Entre os textos coletados, foi encontrado um padrão de abstract, que servirá de base para a análise, baseada no estudo de Ramos e Santos (2006).

- Características do contexto de cultura e de situação

Trata-se de um gênero muito conhecido no meio acadêmico e que tem têm como propósito resumir o artigo, divulgar material científico na área, dar visibilidade à pesquisa e atualizar os leitores da área sobre o desenvolvimento científico. As relações entre os participantes são dadas pelo autor cientista-pesquisador que escreve para a sua comunidade. De acordo com Ramos e Santos (2006), as relações podem ser simétricas (cientista-cientista) e assimétricas (cientista-aluno). O grau de distanciamento entre os participantes é alto e a linguagem é formal. Os textos são produzidos em contextos de pesquisa, como universidades e centros de pesquisas. Eles podem ser escritos em grupos ou individualmente e são veiculados na Língua Inglesa, neste caso, língua não-nativa dos autores. Nos textos podemos saber aonde foram publicados, local, data e filiação dos autores. $\mathrm{O}$ abstract circula juntamente com o artigo na íntegra em periódicos impressos ou eletrônicos. A relevância para a audiência é tomar ciência do que acontece dentro da comunidade acadêmica, já que esse texto é um meio de divulgação científica dos desenvolvimentos da área de estudo. Porém, a relevância pode variar conforme o objetivo do leitor, assim como o assunto varia, mas de maneira geral são resumos de artigos de pesquisa na área de Engenharia Florestal.

- Organização retórica e Características léxico-gramaticais

Seguindo a proposta de Santos (1996) apud Ramos e Santos (2006), na estrutura temos cinco movimentos que compõe o abstract de artigos de pesquisa. São os 
movimentos que tem a função de carregar os conteúdos. Baseada nas autoras, apresento abaixo os movimentos encontrados em um exemplar do gênero ${ }^{7}$. Algumas das características léxico-gramaticais encontradas nos movimentos, tais como as palavras ou expressões referentes ao conteúdo, e os tempos verbais foram grifados na tabela abaixo:

\begin{tabular}{|c|c|c|}
\hline Divisão & Função Comunicativa & Texto \\
\hline Objetivo & $\begin{array}{l}\text { Movimento 1: a pesquisa é } \\
\text { apresentada através da } \\
\text { descrição de seu propósito }\end{array}$ & $\begin{array}{l}\text { This work aimed to evaluate a } \\
\text { forwarder, technically and } \\
\text { economically, under three eucalyptus } \\
\text { forest harvest subsystems. }\end{array}$ \\
\hline Método & $\begin{array}{l}\text { Movimento } \quad 2: \quad r \quad \text { a } \\
\text { metodologia utilizada no } \\
\text { estudo é descrita. }\end{array}$ & $\begin{array}{l}\text { The technical analysis included a } \\
\text { productivity and motion and time } \\
\text { study. The economic analysis included } \\
\text { the parameters operational cost, } \\
\text { production cost and energy } \\
\text { consumption. }\end{array}$ \\
\hline Discussão & $\begin{array}{l}\text { Movimento 3: discussão } \\
\text { dos resultados. }\end{array}$ & $\begin{array}{l}\text { Loading consumed more than } 80 \% \text { of } \\
\text { the total cycle time in the subsystems } \\
\text { studied. The average travel distance } \\
\text { influenced unloaded and loaded } \\
\text { travel times. The forwarder's } \\
\text { operational cost was US } \$ 60.70 / \mathrm{ha} \text {. }\end{array}$ \\
\hline Resultados & $\begin{array}{l}\text { Movimento 4: o autor } \\
\text { concluí a descrição de sua } \\
\text { pesquisa apresentando os } \\
\text { resultados finais. }\end{array}$ & $\begin{array}{l}\text { The forwarder production costs were } \\
\text { US\$ } 1.74,1.53 \text { and } 2.51 / \mathrm{m}^{3} \mathrm{cc} \text {, in } \\
\text { subsystems } 1,2 \text { and } 3 \text {, respectively. } \\
\text { The energy consumption rates were } \\
2.76 ; 2.42 \text { and } 3.98 \mathrm{~g} / \mathrm{kW}^{*} \mathrm{~m}^{3} \mathrm{cc} \text {, in } \\
\text { subsystems } 1,2 \text { and } 3 \text {, respectively }\end{array}$ \\
\hline \multicolumn{3}{|l|}{ Legenda: } \\
\hline $\begin{array}{l}\text { Grifo: Palavro } \\
\text { Itálico: Carac } \\
\text { Negrito: Tem }\end{array}$ & $\begin{array}{l}\text { expressões referentes ao con } \\
\text { icas léxico-gramaticais encor } \\
\text { erbais. }\end{array}$ & $\begin{array}{l}\text { eúdo; } \\
\text { tradas nos movimentos; e }\end{array}$ \\
\hline
\end{tabular}

\section{Quadro 1: Movimentos dos abstracts.}

\footnotetext{
${ }^{7}$ Abstract "Análise técnica e econômica do forwarder em três subsistemas de colheita de florestas de eucalipto" de Luciano José Minette, Fábio Murilo Tieghi Moreira, Amaury Paulo de Souza, Carlos Cardoso Machado e Kátia Regina Silva. Rev. Árvore vol.28 no.1 Viçosa Jan./Feb. 2004.
} 
A possibilidade de não apresentar uma conclusão, faz com que o autor finalize apenas com os resultados da pesquisa, e o texto não traz as escolhas léxico-gramaticais geralmente utilizadas para a conclusão, como por exemplo: this research concludes that... ou the conclusion is....

Vale ressaltar que de acordo com a proposta de gênero, discutida anteriormente nesse artigo, não vemos mais o texto isolado de seus contextos de cultura, de situação, de seu propósito comunicativo, de seus participantes e da sua comunidade discursiva. Acredito que a discussão desses aspectos pode contribuir nas aulas de compreensão e, também, na de produção escrita.

\section{Apresentação das propostas de atividades}

A implementação de gêneros em sala de aula é importante e pode ser feita de vários modos, porém, neste trabalho, escolho utilizar como base a proposta Ramos (2004), que objetiva: conscientizar o aluno do propósito e da estrutura textual dos diferentes gêneros, características lingüísticas, contextuais e sócio-culturais; criar condições de compreensão crítica dos gêneros; proporcionar o conhecimento de formas textuais e conteúdos que constroem os gêneros; e fazer com que o aluno identifique as estratégias necessárias para usar essas características na sua própria produção. Ressalto que, apesar de não ser foco deste trabalho, a produção textual foi requerida, como se pode ver no final da unidade didática em anexo, por acreditar que a produção do aluno pode ser utilizada para verificar se as tarefas propostas contribuíram para o entendimento do gênero estudado. Pretendo, com base nessas produções, realizar um estudo futuro.

A autora propõe uma aplicação, desenvolvida em três fases: apresentação, detalhamento e aplicação. A proposta visa a progressão de conteúdo, por meio de uma abordagem que parte da exposição geral do conteúdo para a específica, de forma espiralada, ou seja, a apresentação de itens novos com retomada dos pontos já apresentados, para fazer, no final, uma retomada desse aprendizado como apropriação individual do abstract. Apresentarei, a seguir, cada fase da proposta de Ramos (2004) juntamente com a proposta das atividades realizadas baseadas nas concepções tratadas nesse artigo. Essas atividades se encontram em anexo.

\section{- Apresentação}

A primeira fase visa tanto à criação de condições para que o gênero a ser trabalhado seja observado sob uma perspectiva ampla, sua contextualização, sob foco do 
contexto de situação e de cultura, quanto à exploração de dois conceitos: a conscientização e a familiarização com o gênero. A conscientização evoca sensibilização, tomada de consciência, na medida em que evidencia aspectos do contexto de situação e de cultura. A familiarização é entendida pela autora como a identificação do conhecimento que o aluno já tem do gênero em questão e, se for necessário, a disponibilização às informações necessárias que ele ainda não possui sobre determinado gênero. É interessante possibilitar a exposição do aluno a vários exemplares de um mesmo gênero, para que ele possa perceber similaridades e diferenças. Dessa forma, as atividades propostas, nessa fase, têm por objetivo a criação dessas condições, como mostra a tarefa $\mathrm{n}^{\circ} 1$ em anexo. Essa tarefa favorece o levantamento de quanto o aluno conhece sobre o gênero, possibilitando tanto um trabalho de familiarização como de conscientização. A proposta de discussão convida os alunos à reflexão e ao compartilhamento de informações. Já a tarefa $n^{\circ} 2$, em anexo, propõe um trabalho de conscientização das partes que compõe o gênero, levando o aluno a identificá-las para depois trabalhar sua organização retórica na próxima fase detalhamento.

\section{- Detalhamento}

Nesta segunda fase, o foco principal é o trabalho com a organização retórica dos textos e suas características léxico-gramaticais, com o objetivo de conduzir o aluno a usar e vivenciar na prática esses componentes, fornecer condições satisfatórias para a compreensão/produção geral e detalhada dos textos, explorar a função discursiva e os componentes léxico-gramaticais particulares do gênero em pauta, compreender os significados e a relação entre um texto e seu contexto de situação. As tarefas, em anexo, $\mathrm{n}^{\circ}$ 3 e 4 exploram a identificação e o reconhecimento da organização retórica de um abstract. A tarefa 5 enfoca as características léxico-gramaticais e propõe a exploração e o entendimento dos usos e das escolhas feitas pelos autores. Além disso, as três tarefas citadas trabalham as diferenças e as semelhanças que podem ser encontradas em um mesmo gênero.

\section{- $\quad$ Aplicação}

$\mathrm{Na}$ última fase, espera-se que o aluno consolide sua aprendizagem e se aproprie do gênero abstract. Nesse momento, os alunos articulam o trabalho feito nas duas fases anteriores. O objetivo é fazer o aluno trabalhar com o gênero como um todo, reintegrando os vários conhecimentos que veio adquirindo. Há duas etapas que compõe essa fase: a consolidação e a apropriação. A primeira tem como objetivo retomar a idéia e o conceito do todo, fornecendo a possibilidade de consolidar o gênero através de atividades 
diversas com este (como a tarefa $\mathrm{n}^{\circ} 6$, em anexo). A segunda, busca fazer a transferência do gênero para situações da vida real, expondo o aluno a várias situações em que o gênero é utilizado e, também, a produção efetiva do gênero pelo aluno (como a tarefa $n^{\circ} 7$ ). É nessa fase que o aluno precisa explorar seus conhecimentos para poder alcançar eficácia na sua própria produção, aprendendo a fazer isso preocupado com as possibilidades das regras e convenções genéricas. No anexo, as tarefas que exemplificam essa fase pressupõe que o aluno já conheça o gênero em questão. O objetivo, nesta fase, é que o aluno explore o que aprendeu nas fases anteriores e nas leituras de sua área para produzir o gênero trabalhado.

\section{Considerações finais}

Este trabalho teve como objetivo apresentar, dentro das concepções de gênero e tarefa, uma proposta de unidade em um curso de língua estrangeira para fins específicos. De acordo com Ramos e Santos (2004), esse novo jeito de conceber gênero acarreta novos jeitos de ensinar e aprender. A realização desse artigo foi uma grande experiência para mim, como professora e pesquisadora, pois tive a oportunidade de refletir sobre o ensino da língua inglesa, mais especificamente, o desenvolvimento de materiais para o ensino de língua para fins específicos. O artigo, em especial, a proposta de gênero me fez refletir na concepção do texto não mais como algo isolado, mas com o seu propósito comunicativo, bem como o seu contexto de situação e de cultura, seus participantes e sua comunidade discursiva. $\mathrm{O}$ ensino baseado em tarefas permitiu, junto com a concepção de gênero, o foco no contexto, na linguagem em uso, incorporando um propósito comunicativo autêntico, requerendo dos alunos funções como usuários da língua, envolvendo-os nas atividades de mundo real. Como explica Swales (1990), o melhor veículo para se ensinar gêneros é a tarefa. Esta proposta visa contribuir para os estudos sobre gênero e para o desenvolvimento de materiais para o ensino de línguas para fins específicos.

\section{Referências}

BHATIA, V. K. Analyzing genre: language use in professional settings. Longman, 1993.

BHATIA, V. K. Introduction: genre analysis and world Englishes. Special Issue on genre analysis and world englishes, 1997. V. 16, n. 1.p. 313-319. 
EGGINS, S. An introduction to functional linguistics. Pinter Publishers, 1994.

EGGINS, S. \& MARTIN, J. R. Genres and registers of discourse. IN: T.A. van DIJK (ed.) Discourse as structure and process - Discourse Studies, a multidisciplinary introduction. Sage Publications, 1997.

ELLIS, R.. Task-based language learning and teaching. Oxford University Press, 2003.

HALLIDAY, M. A. K. \& HASAN, R. Language, context and text: aspects of language in a social-semiotic perspective. Oxford University Press, 1989.

LEFFA, V. J. Como produzir materiais para o ensino de línguas. In: LEFFA, V. J. (org.). Produção de materiais de ensino - teoria e prática. EDUCAT, 2003.

MARTIN, J.R. Context: register, genre and ideology. IN: English text - systems and structure. John Benjamins Publishing Company, 1992.

NUNAN, D. Designing Tasks for the Communicative Classroom. Cambridge Univeristy Press, 1989.

RAMOS, R. C. G. \& SANTOS, V. B. M. P. O conceito de gênero - Inglês instrumental para o sistema de educação profissional de nível técnico. Curso online. PUCSP/VITAE, 2004. Disponível em http://cogeae.dialdata.com.br/999/1/1/midiateca/U2P3T5generos.pdf. (Acessado em: 31/05/2012)

RAMOS, R. C. G. Gêneros textuais: proposta de aplicação $m$ cursos de língua estrangeira para fins específicos. The ESPecialist, 2004. v. 24, n. 2.p.107-129.

RAMOS, R. C. G. \& SANTOS, V. B. M. P. Operationalizing genre and register to feed genre-based materials preparation for Brazilian English teachers. Comunicação apresentada no Anais eletrônicos do 33 $^{\text {rd }}$. ISFC, São Paulo-SP, 2006.

SANTOS, M. B. The textual organization of research paper abstracts in Applied Linguistics. Text, 1996. 16/4: p. 481-499.

SWALES, J. M. Genre analysis - English in academic and research settings. Cambridge University Press, 1990.

TOMLINSON, B. Materials development in language teaching. Cambridge University Press, 1998.

VIAN JR., O. O ensino de inglês instrumental para negócios, a lingüística sistêmicofuncional e a teoria de gêneros/registro. The ESPecialist, 2003. v. 24.1:1-16.

WILLIS \& WILLIS. Task-based language learning. In: The Cambridge guide to teaching English to speakers of other languages. Cambridge University Press, 1996. p. 173-179. 


\section{Anexos}

Trataremos, nesta unidade, um gênero muito conhecido na comunidade acadêmica, o abstract. Vamos conversar um pouco sobre ele, entende-lo e compreende-lo melhor.

1. Perguntas de reflexão:

a) Quem escreve abstracts?

b) Quem lê abstracts?

c) Por que as pessoas escrevem abstracts? Qual é o objetivo comunicativo desse gênero?

d) Por que as pessoas lêem abstracts?

e) Aonde um abstract pode ser encontrado?

f) Como ele é veiculado?

g) Que tipos de informações podem ser encontradas nesse gênero?

h) Qual o papel do abstract na comunidade acadêmica?

2. Leia atentamente o texto abaixo:

FLORESTA, Curitiba, PR, v. 37, n. 1, jan./abr. 2007.

Timofeiczyk Jr., R. et al.

\section{INDICADORES ECONÔMICO-FINANCEIROS DO MANEJO DE BAIXO IMPACTO EM FLORESTAS TROPICAIS - UM ESTUDO DE CASO}

Romano Timofeiczyk Júnior*, Ricardo Berger*, Roberto Antonio Ticle de Melo e Sousa**, Versides Sebastião de Moraes e Silva** *Eng. Florestal, Dr., Depto. de Economia Rural e Extensão, UFPR - romano@floresta.ufpr.br berger@floresta.ufpr.br

**Eng. Florestal, Dr., Depto. de Engenharia Florestal, UFMT - ratms@ terra.com.br versides@uol.com.br

Recebido para publicação: 14/10/2005 - Aceito para publicação: 12/07/2006

\section{Abstract}

1Economic and financial indicators of low impact management in the tropical forest - a case study. 
2This work had as objective to analyze the economic and financial indicators of low impact

3 management in the tropical forest in two production cycles. Data were obtained from a farm located

4in the municipality of Marcelândia, State of Mato Grosso, which harvests a tropical forest and sells

5 timber for wooden industries established in its region. The economic and or financial indicators

6showed to be lower in all analyzed situations, with the minimum values being observed in the first

7cycle. However, in the second cycle those values have increased and reached a maximum.

8Disregarding investment in land, a significant increase was observed in the indicators for all situations

9in the first cycle, with faster increments in the second cycle. Forest species destined to veneer

10production presented negative economic indicators for the first cycle, and a small increment in the

11second cycle. On the other hand, species used by sawmills presented greater positive values, but still

12lesser than the ideal, considering as reference a minimal rate of interest of $12 \%$ per year. From all

13species, itaúba presented the greatest profitability.

Keywords: Low impact management costs; tropical forest; structure of costs.

Agora, identifique quais são os componentes do gênero marcando um X entre as opções abaixo:
○ Introdução
○ Conclusão
- Filiação acadêmica
○ Avaliação do livro
- Resultados de pesquisa
○ Key words
- Metodologia e procedimentos
- Nome do autor do livro
○ Discussão dos resultados
- Resumo do livro
○ Referência bibliográfica
- Organização do livro

3. Leia novamente o texto do exercício anterior e identifique as diferentes partes de um abstract. 


\begin{tabular}{|l|l|}
\hline Partes do abstract & Situe no texto (parágrafo e/ou linha) \\
\hline & \\
\hline & \\
\hline & \\
\hline & \\
\hline
\end{tabular}

Agora, leia este outro abstract e complete a mesma tabela que você completou antes referente a ele:

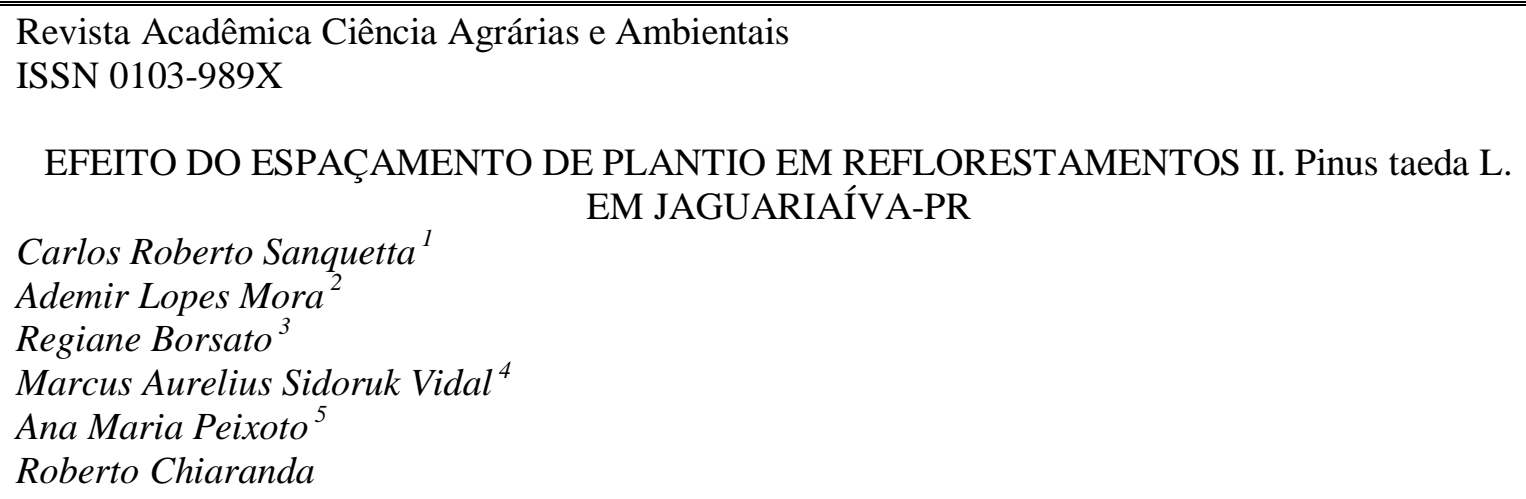

1Abstract - An experiment was carried out in Jaguariaíva, Paraná, aiming at determining the 2effects of various planting densities on the growth of loblolly pine experimental stands by 3 monitoring the following. Variables: average total height, dominant height, DBH (diameter 4at breast height), transversal area, basal area, average individual volume, and volume per 5 hectare. The Bartlett's test was used to test the homogeneity of variance for all variables. 6The results showed that height was not considerably affected by density at 42 and 837 months of age. Statiscally different results, although rather small, were observed at 8intermediate ages. DBH was strongly affected by density and difference among treatments 9were more noticeable with increasing age. As spacing increased, basal area per hectare 10decreased at all ages, whereas average transversal area increased with spacing at all 11ages. Volume per hectare differed among treatments, showing a trend toward increase in 12 volume at higher density stands, although a definite pattern was not clearly detected 13among different stand ages. Results for mean tree volume followed the same trend as 14those for individual basal area. These findings show that plant height is not markedly 15 affected by tree density, while variables related to dbh are.

Key words : Stands, Pine, Forest productivity

1 Engo Florestal, M.Sc., Ph.D., Professor do Curso de Pós-Graduação em Eng. Florestal - SCA UFPR - Bolsista do CNPq.

2 Engo Florestal , M.Sc., Estudante de Doutorado do Curso de Pós-Graduação em Engenharia Florestal - SCA - UFPR.

3 Engo Florestal, Estudante de Mestrado do Curso de Pós-Graduação em Engenharia Florestal SCA - UFPR

4 Eng. Florestal, Estudante de Mestrado do Curso de Pós-Graduação em Engenharia Florestal SCA - UFPR

5 Eng. Florestal, Estudante de Mestrado do Curso de Pós-Graduação em Engenharia Florestal SCA - UFPR

6 Eng. Florestal, Estudante de Mestrado do Curso de Pós-Graduação em Engenharia Florestal -

SCA - UFPR 


\begin{tabular}{|l|l|}
\hline Partes do abstract & Situe no texto (parágrafo e/ou linha) \\
\hline & \\
\hline & \\
\hline & \\
\hline & \\
\hline & \\
\hline
\end{tabular}

4. Compare as diferenças e semelhanças entre os dois abstracts do exercício anterior.

\begin{tabular}{|l|l|}
\hline Semelhanças & Diferenças \\
\hline & \\
& \\
\hline
\end{tabular}

Discuta com a sua turma:

- Quais são as semelhanças encontradas nos dois abstracts? E as diferenças?

- Se você estivesse interessado (a) em saber os procedimentos utilizados na pesquisa, em que parte você procuraria? Por quê?

- E se o seu interesse fosse no problema da pesquisa, em que parte você procuraria? Por quê?

- E se o seu interesse fosse nos resultados encontrados, em qual parte você procuraria? Por quê?

5. No exercício $\mathbf{n}^{\circ} 3$, você localizou partes de dois abstracts. Para localizar essas partes, provavelmente você procurou elementos lingüísticos e não lingüísticos que o ajudaram nessa busca. Quais são os elementos lingüísticos que você considerou importantes para cada parte dos textos? Complete a tabela e discuta com a classe. (Caso não haja a "parte" no (s) texto (s) marque um traço e coloque a parte existente nas células em branco). 


\begin{tabular}{|l|l|l|}
\hline Parte & Abstract 1 & Abstract 2 \\
\hline Objetivo & & \\
\hline Método & & \\
\hline Discussão & & \\
\hline Resultados & & \\
\hline & & \\
\hline & & \\
\hline
\end{tabular}

6. Cada aluno fica encarregado de trazer um abstract no próximo encontro. Em pequenos grupos, selecionem diferentes tipos de abstracts trazidos pelos colegas e verifiquem a estrutura organizacional e os aspectos léxico-gramaticais trabalhados anteriormente. Os grupos deverão fazer uma discussão comparando os resultados (os aspectos semelhantes e os diferentes) dos abstracts trabalhados.

7. Em casa, cada aluno esboçará um abstract de uma pesquisa que realizou ou que está realizando na área. Na aula, o professor atenderá as principais dúvidas encorajando-os a produzirem seus próprios abstracts. 